\title{
Adrenergic receptor-mediated modulation of striatal firing
}

patterns

\author{
Hiroyuki Ohta ${ }^{1}$, Yu Kohno², Masashi Arake ${ }^{3,4}$, Risa Tamura1, Suguru Yukawa ${ }^{5}$, Yoshiaki \\ Sato $^{5}$, Yuji Morimoto ${ }^{3}$, Yasuhiro Nishida ${ }^{1}$ and Hiromu Yawo ${ }^{6}$
}

${ }^{1}$ Department of Physiology, National Defense Medical College, Saitama, Japan

${ }^{2}$ School of Science and Engineering, Tokyo Denki University, Saitama, Japan

${ }^{3}$ Department of Integrative Physiology and Bio-Nano Medicine, National Defense Medical College

${ }^{4}$ Aeromedical Laboratory, Japan Air Self Defense Force, Tokyo, Japan

${ }^{5}$ Teikyo Heisei University, Tokyo, Japan

${ }^{6}$ Department of Developmental Biology and Neuroscience, Tohoku University Graduate School of

Life Sciences and JST, CREST, Sendai, Japan, Tohoku University Basic and Translational Research Centre for Global Brain Science, Sendai, Japan, Center for Neuroscience, Tohoku University Graduate School of Medicine, Sendai, Japan

Correspondence to: Hiroyuki Ohta (ohta@ndmc.ac.jp, 359-8513, Namiki 3-2, Tokorozawa, Saitama, Japan) 


\section{Abstract}

Although noradrenaline and adrenaline are some of the most important neurotransmitters in the central nervous system, the effects of noradrenergic/adrenergic modulation on the striatum have not been determined. In order to explore the effects of adrenergic receptor (AR) agonists on the striatal firing patterns, we used optogenetic methods which can induce continuous firings. We employed transgenic rats expressing channelrhodopsin-2 (ChR2) in neurons. The medium spiny neuron showed a slow rising depolarization during the 1-sec long optogenetic striatal photostimulation and a residual potential with 8.6-sec half-life decay after the photostimulation. As a result of the residual potential, five repetitive 1-sec long photostimulations with 20 -sec onset intervals cumulatively increased the number of spikes. This 'firing increment', possibly relating to the timing control function of the striatum, was used to evaluate the AR modulation. The $\beta$-AR agonist isoproterenol decreased the firing increment between the 1st and 5th stimulation cycles, while the $\alpha_{1}$-AR agonist phenylephrine enhanced the firing increment. Isoproterenol and adrenaline increased the early phase (0-0.5 sec of the photostimulation) firing response. This adrenergic modulation was inhibited by the $\beta$-antagonist propranolol. Conversely, phenylephrine and noradrenaline reduced the early phase response. $\beta$-ARs and $\alpha_{1}$-ARs work in opposition controlling the striatal firing initiation and the firing increment.

$(200 / 200)$ 


\section{Introduction}

Noradrenaline and adrenaline are some of the most important neurotransmitters in the nervous system. However, the effects of noradrenergic/adrenergic modulation on the striatum have not been explored due to a lack of evidence of direct noradrenergic/adrenergic neuron projections into the striatum (Baldo et al., 2003; Berridge and Waterhouse, 2003; Berridge et al., 1997; Jones and Yang, 1985; Swanson and Hartman, 1975). Nonetheless, the presence of noradrenaline in the striatum is firmly established. Striatal noradrenaline levels in freely moving rodents immediately increase responding to unconditioned stimuli (handling and novelty) (Cenci et al., 1992; Ihalainen et al., 1999) and repetitive locus coeruleus (LC) electrical stimulations (Devoto et al., 2005). Abundant adrenergic receptor (AR) expression is confirmed in the striatum. While $\beta$-ARs are primarily found on striatal postsynaptic membranes and cell bodies (Hara et al., 2010; Nicholas et al., 1996; Paschalis et al., 2009; Pisani et al., 2003), $\alpha_{1}$-ARs are found on pre-synaptic terminals in the striatum (Rommelfanger et al., 2009). On the cellular level, although there exists a report of AR-dependent DARPP-32 phosphorylation in the striatum (Hara et al., 2010), there are no reports of how AR ligands modulate striatal electrical properties. In order to inquire into how noradrenaline and adrenaline modulate the striatal functions, we measured the adrenergic modulation on the striatal firing pattern.

A good example of the importance of adrenergic modulation can be seen in Parkinson's disease (PD). Noradrenergic and adrenergic changes have come to be regarded as important factors of PD symptoms (Delaville et al., 2011; LeWitt, 2012; Ostock and Bishop, 2014). 
Although the primary known cause of PD symptoms is the striatal dopamine level reduction induced by the loss of dopaminergic neurons in the substantia nigra ( $\mathrm{SN}$ ), there is a considerable loss of noradrenergic neurons in LC as well (Braak et al., 2003; Halliday et al., 2006; Zarow et al., 2003). Moreover, the pathological changes of LC neurons and melanized projecting neurons in the medulla oblongata precede the SN lesions (Braak et al., 2003). In addition, the noradrenaline loss produces more profound motor deficits than dopaminergic deprivation (Pifl et al., 2013; Rommelfanger et al., 2007). Clinically, it is well-known that $\beta$-agonists aggravate tremors in PD patients (Constas, 1962) and $\beta$-antagonists alleviate the tremors (Crosby et al., 2003; Foster et al., 1984). $\beta$-antagonists are also effective for reducing dyskinesia in PD patients (Carpentier et al., 1996). Knowing noradrenergic/adrenergic modulation on striatal activity is crucial in understanding PD pathology and the drug treatment mechanisms (Delaville et al., 2011; LeWitt, 2012; Ostock and Bishop, 2014).

Medium spiny neurons (MSNs) in the striatum start from a deeply hyperpolarized resting membrane potential, slowly depolarize and show a delayed start of firing during a current injection (Kawaguchi, 1993; Nisenbaum and Wilson, 1995; Planert et al., 2013). The firing pattern during the current injection is basically regular without transient burst firing and the firing rate is relatively low (20-40 Hz maximum in vivo (Gage et al., 2010)). In vivo behavioral studies indicate that MSNs responsive during a task may not be responsive outside of that task (Aldridge and Berridge, 1998; Gardiner and Kitai, 1992). The firing rate is progressively increased after task related cues and events that lead to action selection and reward, and the increased firing continues for several hundred milliseconds to several seconds (Barnes et al., 2011; Gage et al., 2010; Schmitzer-Torbert and Redish, 2008).

Although prolonged activities of MSNs in animals executing a task progressively become 
clear, the adrenergic modulation for firing patterns is still unclear. For the induction of continuous striatal firing, we employed a transgenic rat which expresses channelrhodopsin-2 (ChR2) in neuronal cells (Ji et al., 2012; Tomita et al., 2009). Although patch-clamp is the established intracellular recording method for observing current injection induced firings and electrically stimulated synaptic potentials, it is overly time-consuming for obtaining a large number of stable data, especially from mature, fully-developed animals. Therefore, we used the optogenetic stimulation and multi-unit extracellular spike recording technique in order to comprehensively grasp the firing modulation from hundreds of adult rat neurons.

Surprisingly, repetitive optogenetic stimulation with 20 -sec stimulation onset intervals increased the number of spikes during and after the photostimulation (hereinafter "the firing increment", the definition is in Materials and Methods). Since the firing increment might be related to the striatal function such as learning of action timing control (Doya, 1999; Houk et al., 1995; Jin et al., 2014; Tanaka and Kunimatsu, 2011), we utilized it as an index for the evaluation of adrenergic modulation on the striatum. As a result, we found that $\beta$ - vs. $\alpha_{1}-\mathrm{AR}$ agonists and adrenaline vs. noradrenaline have opposing effects on the striatal firing pattern.

\section{Materials and Methods}

[Ethics statement]

The care and use of all rats in this study was carried out in accordance with institutional ethical guidelines for animal experiments and the safety guidelines for gene manipulation experiments of the National Defense Medical College (Tokorozawa, Saitama, Japan). All experimental procedures were approved by the Animal Research Committee of the National 
Defense Medical College.

[Preparation of striatal slices and solutions]

W-Tg(Thy-1-COP4/YFP*)4Jfhy rats (W-TChR2V4) (Ji et al., 2012; Ohta et al., 2013; Tomita et al., 2009), 60-245 days old, both sexes, were anesthetized with isoflurane and sodium pentobarbital. Ice-cold oxygenated $\left(95 \% \mathrm{O}_{2} / 5 \% \mathrm{CO}_{2}\right)$ cutting solution containing (in mM) $93 \mathrm{NMDG}-\mathrm{Cl}, 30 \mathrm{NaHCO}_{3}, 20 \mathrm{HEPES}, 2.5 \mathrm{KCl}, 10 \mathrm{MgSO}_{4}, 0.5 \mathrm{CaCl}_{2}, 1.2 \mathrm{NaH}_{2} \mathrm{PO}_{4}$, 25 glucose, 2 thiourea, 5 sodium ascorbate and 3 sodium pyruvate (pH 7.4) (Tanaka et al., 2008; Zhao et al., 2011) was transcardially perfused. $500 \mu \mathrm{m}$-thick parasagittal striatal slices were cut using a microslicer (Linear Slicer Pro 7, Dosaka EM, Kyoto, Japan) in the ice-cold cutting solution. Prior to recording, individual slices were incubated at $32-33^{\circ} \mathrm{C}$ for $10-15$ minutes in the cutting solution and then transferred into normal artificial cerebrospinal fluid (aCSF) at $22-24^{\circ} \mathrm{C}$ that consisted of (in $\left.\mathrm{mM}\right) 120 \mathrm{NaCl}, 26 \mathrm{NaHCO}_{3}, 2.5 \mathrm{KCl}, 1 \mathrm{MgCl}_{2}, 2$ $\mathrm{CaCl}_{2}, 1.25 \mathrm{NaH}_{2} \mathrm{PO}_{4}$ and 11 glucose ( $\mathrm{pH}$ 7.4). The slices were constantly perfused with the normal aCSF with $150 \mu \mathrm{M}$ sodium ascorbate at $\sim 2 \mathrm{ml} / \mathrm{min}$ at $25^{\circ} \mathrm{C}$ throughout the recordings.

[Extracellular recording]

A wire tetrode constructed with insulated $18 \mu \mathrm{m}$-diameter nickel-chrome wire (A-M Systems, Washington, USA) was used for multiunit extracellular recordings. Four strands of wire were twisted together and heated to partially melt the insulation. A 4-channel voltage follower pre-amplifier and an amplifier with band-pass filter $(50 \mathrm{~Hz}-3 \mathrm{kHz})$ were used. Data from the tetrode was acquired from the four channels by an A-D/D-A converter (30 kHz/channel, 16 bit; USB-6259BNC, National Instruments, Austin, USA), and stored on a PC 
using Matlab (Mathworks Japan, Tokyo, Japan). Multiunit recording data were processed to isolate spike events by the semi-automatic spike-sorting method (Takekawa et al., 2010). The extracted features were classified, and the classified clusters were then combined or discarded manually using Klusters (Hazan et al., 2006). Raw waves within $20 \mathrm{~ms}$ after the onset of the light pulse were discarded, because the ChR2 photocurrent is not stable during this time window (Ishizuka et al., 2006; Ohta et al., 2013) and photocurrent noise induces sorting errors. Data of fast spiking neurons (> $80 \mathrm{~Hz}$ ), possibly fast spiking interneurons, and tonically active neurons, possibly cholinergic neurons, were discarded.

[Patch clamp]

The striatal neurons were targeted with a upright microscope equipped with a $40 \mathrm{X}$ objective lens using infrared differential interference contrast (AxioSkop 2FS, Zeiss, Jena, Germany). Whole-cell recording pipettes (4-8 M $\Omega$ ) were pulled from borosilicate glass and filled with (mM) $125 \mathrm{~K}$-gluconate, $10 \mathrm{KCl}, 40$ HEPES, $2 \mathrm{MgATP}, 0.3 \mathrm{Na} 3 \mathrm{GTP}$ and 5 Na2-phosphocreatine ( $\mathrm{pH}$ 7.2-7.3). Recordings were made with a CEZ-2400 amplifier (Nihon Kohden, Tokyo, Japan) and the A-D/D-A converter. The liquid junction potential was not corrected.

[Photostimulation]

For the slice extracellular recording experiment, a microscope equipped with a $5 \mathrm{X}$ objective lens was focused on a region of interest in the dorsal striatum area near the corpus callosum. To photostimulate the striatum, a blue LED light source (Luxeon Rebel LXML-PB01-0023, Phillips Lumileds Lighting Company, San Jose, USA) was focused 
through the camera double port with a standard GFP filter cube. The light spot was a $90 \times 90$ $\mu \mathrm{m}$ square and had a light power density of $2.0 \mathrm{~mW} / \mathrm{mm}^{2}$. The pulse intensity of the LED light was precisely controlled by a computer through a custom designed current regulator. The recording electrode tip was placed at the center of the light spot. The striatum was photostimulated 5 times for $1-\mathrm{sec}$ with $20 \mathrm{sec}$ stimulation onset interval. In some cases, the interval was varied from $8^{-} \mathrm{sec}$ to $60^{-}$sec. Unless noted otherwise, results indicate responses for the $20^{-}$sec cycle. We used firing increment as an index to quantify the increased firings after repetitive photostimulation. The firing increment is defined as the difference between the 5th and the 1st photostimulation cycle firing rates. To analyze the mean firing rate and the firing increment during and after photostimulation, three phases were defined: the early phase $(0-0.5 \mathrm{sec}$ after the onset of photostimulation), the late phase $(0.5-1 \mathrm{sec})$ and the post phase $(1-1.5 \mathrm{sec})$ (see Fig. 4A).

[Drugs]

All reagents were from Wako (Osaka, Japan) unless stated otherwise. Reagents used include noradrenaline $\mathrm{HCl}$, (R)-(-)-adrenaline, dl-isoproterenol $\mathrm{HCl}, \mathrm{R}-(-)-$ phenylephrine, propranolol $\mathrm{HCl}, \quad \mathrm{SCH} 23390 \mathrm{HCl}, \mathrm{R}(+)-6-\mathrm{Bromo}-\mathrm{APB}$ hydrobromide (6-Br-APB), 7-nitro-2,3-dioxo-1,4-dihydroquinoxaline-6-carbonitrile $\quad$ (CNQX) and D-(-)-2-amino-5-phosphonopentanoic acid (D-AP5). Adrenaline, SCH23390 and 6-Br-APB were dissolved in dimethyl sulfoxide (DMSO, the final concentration of DMSO was adjusted to below $0.1 \% \mathrm{v} / \mathrm{v})$.

[Statistical analysis] 
Each biological value was expressed as a mean \pm standard error (SEM). Two-way mixed-design analysis of variance (ANOVA) was performed using the phase as the within-subject factor and the drug treatment as the between-subject factor. Dunnett's tests were used for post-hoc comparisons. The analysis was performed using JMP (SAS Institute Japan, Tokyo, Japan). A value of $p<0.05$ was considered to be statistically significant.

\section{Results}

[Residual membrane potential in the medium spiny neuron]

We used a transgenic Wistar W-TChR2V4 rat line, which expresses channelrhodopsin-2 (ChR2) with the Venus fluorescence marker under the regulation of the Thy-1.2 promoter which drives neuron-specific expression of transgenes. ChR2 is expressed in glutamatergic neuron in the cortex. Kuki et al. confirmed $\mathrm{ChR} 2$ expression in $\mathrm{Ca} 2+/$ calmodulin-dependent protein kinase II (CaMKII)-positive neurons in the motor cortex (Kuki et al., 2013). Although cell-type specific ChR2 expression localization in the striatum has not yet been identified by using immunohistochemical methods, Venus fluorescence in the striatum is shown in Fig.1A; and we confirmed the ChR2 oriented response in the medium spiny neuron (MSN) by using electrical methods.

By using whole-cell patch-clamp method on an adult W-TChR2V4 rat MSN (Fig. 1B), we measured the membrane potential changes during and after the LED photostimulation to the striatum. MSNs were identified by: (1) a deep (approx. $-80 \mathrm{mV}$ ) resting membrane potential, (2) a regular spike pattern in response to applied current pulses, (3) long latency to firing of 
the first spike during a current pulse, (4) absence of a voltage "sag" during hyperpolarization (Fig. 1C)(Kawaguchi, 1993; Nisenbaum and Wilson, 1995; Planert et al., 2013). The mean resting membrane potential was $-82.4 \pm 0.8 \mathrm{mV}(\mathrm{n}=9)$. The 1 -sec-long photostimulation induced a slow depolarization and a double exponential decay with a $1.0-\mathrm{sec}$ (fast) and an 8.6-sec (slow) half-life (Fig. 1D). We also observed an increase of the number of action potentials after repetitive photostimulations with 8 -sec stimulation onset interval (8-sec cycle) (Fig. 1E and 1F). The residual decay seemed to contribute to the up-regulation of the basal membrane potential of the next cycle (Fig. 1E; dotted line is the resting membrane potential before the photostimulation). The mean increase of action potential between the 5 th and 1 st cycle was $5.75 \pm 1.59(\mathrm{n}=9)($ Fig. 1F). In contrast, 8-sec cycle repetitive current injections (200 pA) were unable to induce any residual depolarization after the current injections (Fig. 1G). The 1-sec long 8-sec cycle repetitive minimal current injections (100-300 pA) evoking a few action potentials (1-9 spikes) in the 1st cycle did not show a marked increase of the number of action potentials (Fig. $1 \mathrm{H} ;-0.88 \pm 0.48$ spikes, $\mathrm{n}=9$ ).

[Cycle dependency of firing increment]

We extracellularly recorded the striatal firing response to the repetitive photostimulation by a tetrode. Continuous firings during and after 1 -sec long photostimulation were increased by 5 repetitive stimulations on an 8 -sec cycle (Fig. 2).

We sometimes observed high-frequency spiking only during the photostimulation. The spiking was possibly from the fast-spiking neurons (Kawaguchi, 1993; Planert et al., 2010) and we excluded the data from the analysis. We also observed tonic spiking without photostimulation. The tonic spiking was possibly from the cholinergic neurons (Kawaguchi, 
1993). The tonic spiking was silenced after 2-3 1-sec long photostimulations. We also excluded the tonic spiking data from our analysis.

Single unit spike events were isolated (see Materials and Methods) from the multiunit tetrode data recording. We measured the firing increment of five $1-\mathrm{sec}$ long stimulations with 8-60 sec intervals (8-60 sec cycles). In each cycle, the firing rate at each 50-ms time bin for each neuron was calculated and a mean of the firing rate was shown in Fig. 3 (Cycle 1-5). Five $8^{-}, 10^{-}$and $20^{-}$sec cycles induced a positive firing increment in all phases (Fig. 3A-C), while 60-sec cycle photostimulation did not induce an obvious firing increment (Fig. 3D). In order to quantify the firing increment, we calculated mean firing rates of three phases (Fig. 3A: Cycle 1): the early phase $(0-0.5 \mathrm{sec}$ after the onset of photostimulation, blue), the late phase (0.5-1 sec, red) and the post phase (1-1.5 sec, green). In $8^{-}$and 10-sec cycles, the firings linearly increased in the all phases (Fig. 3A and 3B). In a 20-sec cycle, the firing rate differences between the 2 nd and the 5th cycle were smaller than the ones between the 1 st and the 2nd cycle (Fig. 3C). The 20-sec cycle was the maximum stimulation interval which could stably induce the firing increment. We chose the 20 -sec cycle as the default for the assessment of noradrenergic/adrenergic modulation, since it would be more sensitive to the effects of the receptor agonists/antagonists.

[Robustness of the increased firing]

To test the robustness of the firing increase once developed, a conditioning stimulation with five 8-sec cycles was followed by an intermission and a sixth test stimulation (Fig. 4A). In the case of a short intermission, the firing pattern of the sixth cycle was similar to the 
previous one (eg. Fig. 4A middle); however, the number of spikes decreased with a longer intermission (eg. Fig. 4A bottom). Fig. 4B shows the correlation between the post-intermission spike count difference (6th - 1st cycle) and the pre-intermission spike count difference $(5$ th -1 st cycle) for varying intermission lengths $(8-120 \mathrm{sec})$. To evaluate this quantitatively, the ratio of post- to pre-intermission spike count differences was calculated and used to define an index - the remnant (Fig. 4C). The remnant was almost equal to 1 when the intermission was less than or equal to $20 \mathrm{sec}$, and it decreased as a function of time for intermissions longer than $20 \mathrm{sec}$.

[Glutamatergic dependency]

We measured the effects of photostimulation to the glutamatergic fibers on the firing pattern. The blocking of AMPA and NMDA receptors by the bath-application of $20 \mu \mathrm{M}$ CNQX and $50 \mu \mathrm{M}$ D-AP5 induced a significant reduction of the 1st cycle early phase response (Fig.5; CNQX+AP5: $1.99 \pm 1.87$ spike/sec, $n=23$ isolated neurons, $\mathrm{p}<0.005$, CTRL: $9.19 \pm 0.34$ spike/sec, $n=692$ isolated neurons). The 1 st cycle late and post phase responses were not significantly changed (CNQX+AP5: $12.09 \pm 1.78$ spike/sec, $n=23, p=0.99$, CTRL: $9.90 \pm 0.32$ spike/sec in late phase; CNQX+AP5: $7.74 \pm 1.13$ spike/sec, $n=23, p=0.16$, CTRL: $4.75 \pm 0.21$ spike/sec in post phase). This indicates that the photostimulation induced firings in the striatum depend on both the glutamatergic post-synaptic potential and the direct photostimulation on the striatum neurons.

[ $\beta$-AR firing acceleration] 
A bath-application of $50 \mu \mathrm{M}$ isoproterenol, a $\beta$-AR agonist, significantly increased the $1 \mathrm{st}$ cycle early phase firing response (Fig. 6A; isoproterenol: $13.35 \pm 1.15$ spike/sec, $n=61$ isolated neurons, $\mathrm{p}<0.05)$ and significantly decreased the 1 st cycle post phase firing response (isoproterenol: $2.52 \pm 0.69$ spike/sec, $\mathrm{n}=61, \mathrm{p}<0.05$, CTRL: $4.75 \pm 0.21$ spike/sec, $\mathrm{n}=692$ ). Isoproterenol also significantly reduced the late phase firing increment (isoproterenol: 0.07 \pm 1.42 spike/sec, $n=61, \mathrm{p}<0.05$, CTRL: $4.51 \pm 0.42$ spike/sec, $n=692)$. The summary plots of the 1 st cycle firing response and the firing increment are presented in Fig. 8A and 8B.

$20 \mu \mathrm{M}$ adrenaline also significantly increased the 1st cycle early phase firing response (Fig. 6B and 8A; adrenaline: $15.60 \pm 1.52$ spike/sec, $n=35, \mathrm{p}<0.005$ ) while the post phase response was unchanged (adrenaline: $4.97 \pm 0.91$ spike/sec, $n=35, \mathrm{p}=1.00$ ).

Applications of $20 \mu \mathrm{M}$ adrenaline with $20 \mu \mathrm{M}$ propranolol, a $\beta$-AR antagonist, did not show any significant enhancement of the 1st cycle early phase firing (Fig. 6C and 8A; adrenaline+propranolol, $6.57 \pm 1.43$ spike/sec, $n=39, p=0.75)$.

[ $\alpha_{1}-\mathrm{AR}$ firing delay and firing increment]

A bath-application of $30 \mu \mathrm{M}$ phenylephrine, an $\alpha_{1}$-AR agonist, decreased the 1 st cycle early firing response (Fig. 7A and 8A; phenylephrine: $5.59 \pm 0.94$ spike/sec, $n=92, p<0.01$ ). Moreover, the late and post phase firing increments were significantly increased (Fig. 7A and 8B; phenylephrine: $8.20 \pm 1.15$ spike/sec, $n=92, p<0$. 05, CTRL: $4.51 \pm 0.42$ spike/sec, $n=692$ in late phase, phenylephrine: $5.22 \pm 0.75$ spike/sec, $n=92, p<0.0005$, CTRL: $1.76 \pm 0.28$ spike/sec, $\mathrm{n}=692$ in post phase). 
Similarly, $50 \mu \mathrm{M}$ noradrenaline decreased the 1st cycle early phase firing response (Fig. 7B and 8A; noradrenaline: $4.18 \pm 0.83$ spike/sec, $n=118, \mathrm{p}<0.0001)$.

\section{Discussion}

We found that $\beta$-AR vs. $\alpha_{1}-\mathrm{AR}$ agonists and adrenaline vs. noradrenaline have opposing effects on striatal firing. Photostimulation to the W-TChR2V4 rat striatum induced a slow decaying depolarization (Fig. 1D). The 8-20 sec cycle repetitive photostimulation induced a firing increment (Fig. 1C, 2 and 3) and residual effects after a 20-sec intermission (Fig. 4C). We utilized these phenomena to evaluate the adrenergic receptor modulation on the striatum. As a result, $\beta$-agonist isoproterenol and adrenaline increased the early phase firing response and isoproterenol decreased the post phase firing response (Fig. 6A, 6B and 8A), while $\alpha 1$-agonist phenylephrine and noradrenaline decreased the early phase firing response (Fig. 7A, 7B and 8A). Moreover, isoproterenol inhibited the late phase firing increment (Fig. 6A and $8 \mathrm{~B}$ ), while phenylephrine increased the late and post phase firing increment (Fig. 7A and 8B). This implies that the activation pattern of adrenergic receptors may influence timing-control related striatal processes: motor control and action sequence learning by reward (Doya, 1999; Houk et al., 1995; Jin et al., 2014), as well as the pathophysiology of the PD symptoms: movement and executive function disorders (Cameron et al., 2010; Fogelson et al., 2011).

Although Venus fluorescence with ChR2 was broadly observed in the striatum (Fig. 1A), the precise cell-type specific ChR2 expression in the striatum has not yet been identified. 
However, we confirmed the ChR2 oriented response in MSN by using the patch-clamp method (Fig. 1B-E). The blocking of AMPA/NMDA receptors revealed that photostimulation to the striatum results in the activation both of the pre-synaptic glutamatergic fibers and post-synaptic direct depolarization (Fig. 5). The post-synaptic direct depolarization possibly comes from dendritic ChR2 activation, because the slow decaying depolarization was observed only after photostimulation (Fig. 1E) but not after current injection (Fig. 1G).

[Slow decay and Firing increment]

Rising depolarization was observed during and decaying depolarization after the photostimulation (Fig. 1D). The slow decrease of the firing rate after photostimulation is also a remarkable characteristic of the striatal neuron (Fig. 1E, 2 and 3), since the cortex pyramidal neurons (data not shown) and the hippocampus CA1 pyramidal neurons (Ohta et al., 2013) show a firing pattern strictly matching the photostimulation duration without any responses after the photostimulation.

In MSNs, prolonged dendritic calcium activation which is correlated to the sustained depolarization "upstate" was reported (Day et al., 2008; Kerr and Plenz, 2004, 2002; Kitano et al., 2002; Plenz and Kitai, 1998; Plotkin et al., 2011; Wilson and Kawaguchi, 1996). It was also reported that depolarizing stimulus upregulates the probability of spike generation after several hundred ms (Mahon et al., 2003, 2000a, 2000b; Onn et al., 1994). However, there were no obvious reports of the sustained continuous firings in slice preparations and the deca-second order sustained mode changes. To this, we discovered the firing increment induced by the 8 - to 20 -sec cycle repetitive stimulations (Fig. 3A-C) and the residual effect after the 20-sec intermission (Fig. 4C). We observed the slow depolarization decay with an 8.6-sec half-life (Fig. 1D) and the accumulation of the residual depolarization after the 
repetitive photostimulations (Fig. 1E). The firing increment and the residual effect are possibly due to a lack of leak channels (e.g. hyperpolarization-activated cyclic nucleotide-gated channel) (Monteggia et al., 2000; Ohta, 2015).

We demonstrated that isoproterenol and adrenaline increase the early phase firing response (Fig. 6A and 6B), while propranolol inhibits the adrenaline induced enhancement of the early phase firing response (Fig. 6C). Although $\beta$-ARs are found on striatal postsynaptic membranes and cell bodies (Hara et al., 2010; Nicholas et al., 1996; Paschalis et al., 2009; Pisani et al., 2003), the precise roles are not known. In the hippocampus, it is reported that the activation of $\beta$-ARs modulate synaptic inputs (Dunwiddie et al., 1992; Katsuki et al., 1997). Since the early phase firing response is related to the glutamatergic inputs (Fig. 5), the $\beta$-ARs could be related to the amplification of the transient synaptic inputs.

Phenylephrine inhibited the early phase firing response and potentiated the late and post phase firing increment (Fig. 7A). Noradrenaline inhibited the early phase firing response (Fig. 7B). These data signify that the $\alpha_{1}-\mathrm{AR}$ affects the striatum by delaying firing initiation and enhancing continued firing. Recently, it has been reported that noradrenaline drives persistent activity in rat prefrontal cortex via $\alpha_{1}$ - and $\alpha_{2}$-ARs (Zhang et al., 2013). Although, at this moment, the precise roles of $\alpha_{1}-\mathrm{AR}$ in the striatum are unknown, the enhancement of the late and post phase firing increment by phenylephrine is consistent with these reports.

Noradrenergic/adrenergic as well as dopaminergic alterations have come to be regarded as important factors of PD symptoms (Delaville et al., 2011; LeWitt, 2012; Ostock and Bishop, 2014). The administration of $\beta$-agonist isoproterenol aggravates tremors in PD patients 
(Constas, 1962). Conversely, it is also thought that $\beta$-antagonist may be of use in controlling the tremors (Crosby et al., 2003). $\beta$-antagonist nadolol appears to be an effective adjunct to dopaminergic and anticholinergic therapy for severe tremors in PD (Foster et al., 1984). $\beta$-antagonist propranolol was claimed to reduce essential tremor (Winkler and Young, 1971) and L-DOPA-induced dyskinesia in PD patients (Carpentier et al., 1996). The antidyskinetic effects of propranolol have also been supported in rat studies (Barnum et al., 2012; Lindenbach et al., 2011). In sum, $\beta$-activation exacerbates while $\beta$-inhibition alleviates these movement disorders.

In PD patients, CSF noradrenaline levels have been found to be decreased (Eldrup et al., 1995; Martignoni et al., 1992). The reduction of noradrenaline concentration in CSF of PD patients is significantly correlated with parkinsonian symptoms: the severity of Hoehn and Yahr's stage, akinesia and freezing of gait (Tohgi et al., 1993). The activation of LC, which contains noradrenergic neurons, may inhibit striatal firings in cat, and L-threo-dihydroxyphenylserine (L-DOPS), anoradrenaline precursor, lifts the inhibition (Hirose et al., 1988). L-DOPS is known to inhibit L-3,4-dihydroxyphenylalanine (L-DOPA) treatment-induced freezing of gait in humans. In dopamine-depleted rats, striatal noradrenaline loss exacerbates L-DOPA-induced dyskinesias (Fulceri et al., 2007). These reports consistently indicate that the recovery of decreased noradrenaline levels is important to control PD related symptoms. On the other hand, CSF adrenaline levels in PD were rarely reported. Tohgi et al. reported the increased levels of adrenaline in PD patients with dementia (Tohgi et al., 1993). Chia et al. also reported the increased adrenaline in L-DOPA-untreated and L-DOPA-withdrawn PD patients (Chia et al., 1993). These reports lead to the suggestion that the noradrenergic (and possibly adrenergic) modulations are important factors in PD. 
To this, the evidence of opposite effects of noradrenaline and adrenaline on the striatal firing initiation (Fig. 6B and 7B) should not be ignored, because the striatum is related to controlling action timing by regulating the firing increase rate (Tanaka and Kunimatsu, 2011). Although the ligand supply source of adrenergic receptors in the striatum is still unknown, it is important to study the modulatory effects of noradrenaline and adrenaline on striatal firings, in order to understand the pathophysiology of PD related timing control deficiency. In summary, the activation of $\beta$-ARs accelerates striatal firing initiation and inhibits the firing increment, while the activation of $\alpha_{1}$-ARs delays striatal firing initiation and enhances the firing increment. These findings provide new insights into fundamental striatal mechanisms and pathomechanisms of striatum oriented disease.

Acknowledgments: We are grateful to S. Kanda, Drs. K. Ooyo and Y. Yamaguchi for great technical assistance, and to D. A. Tyurmin for language assistance. This work was supported by MEXT/JSPS KAKENHI Grant Number 24700200, a grant for Specific Research from National Defense Medical College, Defense Medicine Propulsion Research of the Ministry of Defense, Japan, the Cooperative Research Project Program of RIEC, Tohoku University and the Research Foundation for Opto-Science and Technology, Hamamatsu, Japan.

Author Contributions Statement: H.O., Y.M. and H.Y. conceived the experiment, H.O., R.T., M.A., S.Y., and Y.S. conducted the experiment, H.O., M.A. and Y.K. analyzed the results and H.O, M.A., Y.M., Y.N. and H.Y. wrote the main manuscript text. All authors reviewed the manuscript.

Additional Statement: The authors declare no competing financial interests. 


\section{References}

Aldridge, J.W., Berridge, K.C., 1998. Coding of serial order by neostriatal neurons: a "natural action" approach to movement sequence. J. Neurosci. 18, 2777-87.

Baldo, B.A., Daniel, R.A., Berridge, C.W., Kelley, A.E., 2003. Overlapping distributions of orexin/hypocretin- and dopamine-beta-hydroxylase immunoreactive fibers in rat brain regions mediating arousal, motivation, and stress. J. Comp. Neurol. 464, 220-237.

Barnes, T.D., Mao, J.-B., Hu, D., Kubota, Y., Dreyer, A. a, Stamoulis, C., Brown, E.N., Graybiel, A.M., 2011. Advance cueing produces enhanced action-boundary patterns of spike activity in the sensorimotor striatum. J. Neurophysiol. 105, 1861-1878.

Barnum, C.J., Bhide, N., Lindenbach, D., Surrena, M.A., Goldenberg, A.A., Tignor, S., Klioueva, A., Walters, H., Bishop, C., 2012. Effects of noradrenergic denervation on L-DOPA-induced dyskinesia and its treatment by alpha- and beta-adrenergic receptor antagonists in hemiparkinsonian rats. Pharmacol. Biochem. Behav. 100, 607-615.

Berridge, C.W., Stratford, T.L., Foote, S.L., Kelley, A.E., 1997. Distribution of dopamine beta-hydroxylase-like immunoreactive fibers within the shell subregion of the nucleus accumbens. Synapse 27, 230-241.

Berridge, C.W., Waterhouse, B.D., 2003. The locus coeruleus-noradrenergic system: modulation of behavioral state and state-dependent cognitive processes. Brain Res. Rev. 42, 33-84.

Braak, H., Del Tredici, K., Rüb, U., De Vos, R. a I., Jansen Steur, E.N.H., Braak, E., 2003. Staging of brain pathology related to sporadic Parkinson's disease. Neurobiol. Aging 24, 197-211.

Cameron, I.G.M., Watanabe, M., Pari, G., Munoz, D.P., 2010. Executive impairment in Parkinson's disease: Response automaticity and task switching. Neuropsychologia 48, 1948-1957.

Carpentier, A.F., Bonnet, A.M., Vidailhet, M., Agid, Y., 1996. Improvement of levodopa-induced dyskinesia by propranolol in Parkinson's disease. Neurology 46, 1548-1551. 
Cenci, M. a, Kalén, P., Mandel, R.J., Björklund, a, 1992. Regional differences in the regulation of dopamine and noradrenaline release in medial frontal cortex, nucleus accumbens and caudate-putamen: a microdialysis study in the rat. Brain Res. 581, 217-228.

Chia, L.G., Cheng, F.C., Kuo, J.S., 1993. Monoamines and their metabolites in plasma and lumbar cerebrospinal fluid of Chinese patients with Parkinson's disease. J. Neurol. Sci. 116, 125-134.

Constas, C., 1962. The effects of adrenaline, noradrenaline, and isoprenaline on parkinsonian tremor. J. Neurol. Neurosurg. Psychiatry 25, 116-121.

Crosby, N.J., Deane, K.H.O., Clarke, C.E., 2003. Beta-blocker therapy for tremor in Parkinson's disease. Cochrane Database Syst. Rev. CD003361.

Day, M., Wokosin, D., Plotkin, J.L., Tian, X., Surmeier, D.J., 2008. Differential excitability and modulation of striatal medium spiny neuron dendrites. J. Neurosci. 28, 11603-14.

Delaville, C., Deurwaerdère, P. De, Benazzouz, A., 2011. Noradrenaline and Parkinson's disease. Front. Syst. Neurosci. 5, 31.

Devoto, P., Flore, G., Saba, P., Fà, M., Gessa, G.L., 2005. Stimulation of the locus coeruleus elicits noradrenaline and dopamine release in the medial prefrontal and parietal cortex. J. Neurochem. $92,368-374$.

Doya, K., 1999. What are the computations of the cerebellum, the basal ganglia and the cerebral cortex? Neural Netw. 12, 961-974.

Dunwiddie, T. V, Taylor, M., Heginbotham, L.R., Proctor, W.R., 1992. Long-term increases in excitability in the CA1 region of rat hippocampus induced by beta-adrenergic stimulation: possible mediation by cAMP. J. Neurosci. 12, 506-17.

Eldrup, E., Mogensen, P., Jacobsen, J., Pakkenberg, H., Christensen, N.J., 1995. CSF and plasma concentrations of free norepinephrine, dopamine, 3,4-dihydroxyphenylacetic acid (DOPAC), 
3,4-dihydroxyphenylalanine (DOPA), and epinephrine in Parkinson's disease. Acta Neurol. Scand. 92, 116-121.

Fogelson, N., Fernandez-del-Olmo, M., Santos-Garcia, D., 2011. Contextual processing deficits in Parkinson's disease: The role of the frontostriatal system. Clin. Neurophysiol. 122, 539-545.

Foster, N.L., Newman, R.P., LeWitt, P.A., Gillespie, M.M., Larsen, T.A., Chase, T.N., 1984. Peripheral beta-adrenergic blockade treatment of parkinsonian tremor. Ann. Neurol. 16, 505508.

Fulceri, F., Biagioni, F., Ferrucci, M., Lazzeri, G., Bartalucci, A., Galli, V., Ruggieri, S., Paparelli, A., Fornai, F., 2007. Abnormal involuntary movements (AIMs) following pulsatile dopaminergic stimulation: Severe deterioration and morphological correlates following the loss of locus coeruleus neurons. Brain Res. 1135, 219-229.

Gage, G.J., Stoetzner, C.R., Wiltschko, A.B., Berke, J.D., 2010. Selective Activation of Striatal Fast-Spiking Interneurons during Choice Execution. Neuron 67, 466-479.

Gardiner, T.W., Kitai, S.T., 1992. Single-unit activity in the globus pallidus and neostriatum of the rat during performance of a trained head movement. Exp. brain Res. 88, 517-30.

Halliday, G.M., Del Tredici, K., Braak, H., 2006. Critical appraisal of brain pathology staging related to presymptomatic and symptomatic cases of sporadic Parkinson's disease. J. Neural Transm. Suppl. 99-103.

Hara, M., Fukui, R., Hieda, E., Kuroiwa, M., Bateup, H.S., Kano, T., Greengard, P., Nishi, A., 2010. Role of adrenoceptors in the regulation of dopamine/DARPP-32 signaling in neostriatal neurons. J. Neurochem. 113, 1046-1059.

Hazan, L., Zugaro, M., Buzsáki, G., 2006. Klusters, NeuroScope, NDManager: a free software suite for neurophysiological data processing and visualization. J. Neurosci. Methods, J. Neurosci. Methods \{(Netherlands) $\}$ 155, 207-216. 
Hirose, A., Sasa, M., Ohno, Y., Takaori, S., 1988. Inhibitory effects of L-threo-DOPS, an L-noradrenaline precursor, on locus coeruleus-originating neurons in the caudate nucleus. Jpn. J. Pharmacol. 48, 435-440.

Houk, J.C., Adams, J.L., Barto, A., 1995. A model of how the basal ganglia generate and use neural signals that predict reinforcement. In: Houk, J.C., Davis, J.L., Beiser, D.G. (Eds.), Models of Information Processing in the Basal Ganglia. MIT Press, Cambridge, MA, pp. 249-270.

Ihalainen, J. a., Riekkinen, P., Feenstra, M.G.P., 1999. Comparison of dopamine and noradrenaline release in mouse prefrontal cortex, striatum and hippocampus using microdialysis. Neurosci. Lett. $277,71-74$

Ishizuka, T., Kakuda, M., Araki, R., Yawo, H., 2006. Kinetic evaluation of photosensitivity in genetically engineered neurons expressing green algae light-gated channels. Neurosci. Res. 54, 85-94.

Ji, Z.-G., Ito, S., Honjoh, T., Ohta, H., Ishizuka, T., Fukazawa, Y., Yawo, H., 2012. Light-evoked somatosensory perception of transgenic rats that express channelrhodopsin-2 in dorsal root ganglion cells. PLoS One 7, e32699.

Jin, X., Tecuapetla, F., Costa, R.M., 2014. Basal ganglia subcircuits distinctively encode the parsing and concatenation of action sequences. Nat. Neurosci. 17, 423-430.

Jones, B.E., Yang, T.Z., 1985. The efferent projections from the reticular formation and the locus coeruleus studied by anterograde and retrograde axonal transport in the rat. J. Comp. Neurol. 242, 56-92.

Katsuki, H., Izumi, Y., Zorumski, C.F., 1997. Noradrenergic regulation of synaptic plasticity in the hippocampal CA1 region. J. Neurophysiol. 77, 3013-3020.

Kawaguchi, Y., 1993. Physiological, morphological, and histochemical characterization of three classes of interneurons in rat neostriatum. J. Neurosci. 13, 4908-23. 
Kerr, J.N.D., Plenz, D., 2002. Dendritic calcium encodes striatal neuron output during up-states. J. Neurosci. 22, 1499-512.

Kerr, J.N.D., Plenz, D., 2004. Action potential timing determines dendritic calcium during striatal up-states. J. Neurosci. 24, 877-85.

Kitano, K., Câteau, H., Kaneda, K., Nambu, A., Takada, M., Fukai, T., 2002. Two-state membrane potential transitions of striatal spiny neurons as evidenced by numerical simulations and electrophysiological recordings in awake monkeys. J. Neurosci. 22, RC230.

Kuki, T., Ohshiro, T., Ito, S., Ji, Z.G., Fukazawa, Y., Matsuzaka, Y., Yawo, H., Mushiake, H., 2013. Frequency-dependent entrainment of neocortical slow oscillation to repeated optogenetic stimulation in the anesthetized rat. Neurosci. Res. 75, 35-45.

LeWitt, P.A., 2012. Norepinephrine: the next therapeutics frontier for Parkinson's disease. Transl. Neurodegener. 1, 4.

Lindenbach, D., Ostock, C.Y., Eskow Jaunarajs, K.L., Dupre, K.B., Barnum, C.J., Bhide, N., Bishop, C., 2011. Behavioral and cellular modulation of L-DOPA-induced dyskinesia by beta-adrenoceptor blockade in the 6-hydroxydopamine-lesioned rat. J. Pharmacol. Exp. Ther. $337,755-765$.

Mahon, S., Casassus, G., Mulle, C., Charpier, S., 2003. Spike-dependent intrinsic plasticity increases firing probability in rat striatal neurons in vivo. J. Physiol. 550, 947-59.

Mahon, S., Delord, B., Deniau, J.M., Charpier, S., 2000a. Intrinsic properties of rat striatal output neurones and time-dependent facilitation of cortical inputs in vivo. J. Physiol. 527 Pt 2, 345-54.

Mahon, S., Deniau, J.M., Charpier, S., Delord, B., 2000b. Role of a striatal slowly inactivating potassium current in short-term facilitation of corticostriatal inputs: a computer simulation study. Learn. Mem. 7, 357-62. 
Martignoni, E., Blandini, F., Petraglia, F., Pacchetti, C., Bono, G., Nappi, G., 1992. Cerebrospinal fluid norepinephrine, 3-methoxy-4-hydroxyphenylglycol and neuropeptide Y levels in Parkinson's disease, multiple system atrophy and dementia of the Alzheimer type. J. Neural Transm. - Park. Dis. Dement. Sect. 4, 191-205.

Monteggia, L.M., Eisch, a J., Tang, M.D., Kaczmarek, L.K., Nestler, E.J., 2000. Cloning and localization of the hyperpolarization-activated cyclic nucleotide-gated channel family in rat brain. Brain Res. Mol. Brain Res. 81, 129-39.

Nicholas, A.P., Hökfelt, T., Pieribone, V.A., 1996. The distribution and significance of CNS adrenoceptors examined with in situ hybridization. Trends Pharmacol. Sci. 17, 245-255.

Nisenbaum, E.S., Wilson, C.J., 1995. Potassium currents responsible for inward and outward rectification in rat neostriatal spiny projection neurons. J. Neurosci. 15, 4449-4463.

Ohta, H., 2015. Reevaluation of McCulloch-Pitts-von Neumann's clock. Biosystems 127, 7-13.

Ohta, H., Sakai, S., Ito, S., Ishizuka, T., Fukazawa, Y., Kemuriyama, T., Tandai-Hiruma, M., Mushiake, H., Sato, Y., Yawo, H., Nishida, Y., 2013. Paired stimulation between CA3 and CA1 alters excitability of CA3 in the rat hippocampus. Neurosci. Lett. 534, 182-7.

Onn, S.P., Berger, T.W., Grace, A.A., 1994. Identification and characterization of striatal cell subtypes using in vivo intracellular recording in rats: II. Membrane factors underlying paired-pulse response profiles. Synapse 16, 195-210.

Ostock, C.Y., Bishop, C., 2014. The Role of the Noradrenergic System and Its Receptors in Levodopa-Induced Dyskinesia. In: Fox, S.H., Brotchie, J.M. (Eds.), Levodopa-Induced Dyskinesia in Parkinson's Disease. Springer London, pp. 265-283.

Paschalis, A., Churchill, L., Marina, N., Kasymov, V., Gourine, A., Ackland, G., 2009. Beta1-Adrenoceptor distribution in the rat brain: an immunohistochemical study. Neurosci. Lett. $458,84-88$. 
Pifl, C., Hornykiewicz, O., Blesa, J., Adánez, R., Cavada, C., Obeso, J.A., 2013. Reduced noradrenaline, but not dopamine and serotonin in motor thalamus of the MPTP primate: Relation to severity of Parkinsonism. J. Neurochem. 125, 657-662.

Pisani, A., Bonsi, P., Centonze, D., Martorana, A., Fusco, F., Sancesario, G., Persis, C. De, Bernardi, G., Vergata, T., Santa, F., De Persis, C., Calabresi, P., 2003. Activation of beta1-adrenoceptors excites striatal cholinergic interneurons through a cAMP-dependent, protein kinase-independent pathway. J. Neurosci. 23, 5272-5282.

Planert, H., Berger, T.K., Silberberg, G., 2013. Membrane properties of striatal direct and indirect pathway neurons in mouse and rat slices and their modulation by dopamine. PLoS One 8 , e57054.

Planert, H., Szydlowski, S.N., Hjorth, J.J.J., Grillner, S., Silberberg, G., 2010. Dynamics of Synaptic Transmission between Fast-Spiking Interneurons and Striatal Projection Neurons of the Direct and Indirect Pathways. J. Neurosci. 30, 3499-3507.

Plenz, D., Kitai, S.T., 1998. Up and down states in striatal medium spiny neurons simultaneously recorded with spontaneous activity in fast-spiking interneurons studied in cortex-striatum-substantia nigra organotypic cultures. J. Neurosci. 18, 266-83.

Plotkin, J.L., Day, M., Surmeier, D.J., 2011. Synaptically driven state transitions in distal dendrites of striatal spiny neurons. Nat. Neurosci. $14,881-8$.

Rommelfanger, K.S., Edwards, G.L., Freeman, K.G., Liles, L.C., Miller, G.W., Weinshenker, D., 2007. Norepinephrine loss produces more profound motor deficits than MPTP treatment in mice. Proc. Natl. Acad. Sci. U. S. A. 104, 13804-13809.

Rommelfanger, K.S., Mitrano, D.A., Smith, Y., Weinshenker, D., 2009. Light and electron microscopic localization of alpha-1 adrenergic receptor immunoreactivity in the rat striatum and ventral midbrain. Neuroscience 158, 1530-1540. 
Schmitzer-Torbert, N.C., Redish, A.D., 2008. Task-dependent encoding of space and events by striatal neurons is dependent on neural subtype. Neuroscience 153, 349-60.

Swanson, L.W., Hartman, B.K., 1975. The central adrenergic system. An immunofluorescence study of the location of cell bodies and their efferent connections in the rat utilizing dopamine-beta-hydroxylase as a marker. J. Comp. Neurol. 163, 467-505.

Takekawa, T., Isomura, Y., Fukai, T., 2010. Accurate spike sorting for multi-unit recordings. Eur. J. Neurosci. 31, 263-272.

Tanaka, M., Kunimatsu, J., 2011. Contribution of the central thalamus to the generation of volitional saccades. Eur. J. Neurosci. 33, 2046-57.

Tanaka, Y., Tanaka, Y., Furuta, T., Yanagawa, Y., Kaneko, T., 2008. The effects of cutting solutions on the viability of GABAergic interneurons in cerebral cortical slices of adult mice. J. Neurosci. Methods 171, 118-125.

Tohgi, H., Abe, T., Takahashi, S., Takahashi, J., Nozaki, Y., Ueno, M., Kikuchi, T., 1993. Monoamine metabolism in the cerebrospinal fluid in Parkinson's disease: relationship to clinical symptoms and subsequent therapeutic outcomes. J. Neural Transm. - Park. Dis. Dement. Sect. 5, 17-26.

Tomita, H., Sugano, E., Fukazawa, Y., Isago, H., Sugiyama, Y., Hiroi, T., Ishizuka, T., Mushiake, H., Kato, M., Hirabayashi, M., Shigemoto, R., Yawo, H., Tamai, M., 2009. Visual properties of transgenic rats harboring the channelrhodopsin- 2 gene regulated by the thy-1.2 promoter. PLoS One 4, e7679.

Wilson, C.J., Kawaguchi, Y., 1996. The origins of two-state spontaneous membrane potential fluctuations of neostriatal spiny neurons. J. Neurosci. 16, 2397-410.

Winkler, G.F., Young, R.R., 1971. The control of essential tremor by propranolol. Trans. Am. Neurol. Assoc. 96, 66-68. 
Zarow, C., Lyness, S. a, Mortimer, J. a, Chui, H.C., 2003. Neuronal loss is greater in the locus coeruleus than nucleus basalis and substantia nigra in Alzheimer and Parkinson diseases. Arch. Neurol. 60, 337-341.

Zhang, Z., Cordeiro Matos, S., Jego, S., Adamantidis, A., Séguéla, P., 2013. Norepinephrine Drives Persistent Activity in Prefrontal Cortex via Synergistic $\alpha 1$ and $\alpha 2$ Adrenoceptors. PLoS One 8, e66122.

Zhao, S., Ting, J.T., Atallah, H.E., Qiu, L., Tan, J., Gloss, B., Augustine, G.J., Deisseroth, K., Luo, M., Graybiel, A.M., Feng, G., 2011. Cell type-specific channelrhodopsin-2 transgenic mice for optogenetic dissection of neural circuitry function. Nat. Methods. 


\section{Figure Legends}

Figure 1. Time courses of membrane potential changes of an adult rat striatal medium spiny neuron (MSN). A. Venus fluorescence (green) and the 4',6-diamidino-2-phenylindole (DAPI, blue) in the W-TChR2V4 rat striatum. White scale bar represents $20 \mu \mathrm{m}$. B. Patch-clamped MSN. Black scale bar represents $20 \mu \mathrm{m}$. C. Current injection induced membrane potential changes of MSN. D. 1-sec-long photostimulation induced a slow rising depolarization and a slow decay. The orange line is a best fit double exponential decay curve. E. Repetitive photostimulation over an $8 \mathrm{sec}$ cycle up-regulated the membrane potential baseline and increased the number of spikes. Dotted horizontal lines are the resting membrane potential before the 1st photostimulation (Cycle 1). F. Optogenetically induced firing increase. Five 1-sec-long photostimulations with 8-sec interval increased the number of action potentials. G. Repetitive current injections (200 pA, 1-sec-long, 8-sec interval) did not produce the slow rising depolarization nor the slow decay. H. Minimal repetitive current injections did not increase the number of spikes.

Figure 2. Extracellularly recorded striatum firing responses to striatum photostimulation. Five 1-sec long photostimulations with 8-sec onset interval (cycle) increased the number of firings around the offset of the photostimulations.

Figure 3. Photostimulation cycle length dependency of striatal firing increment. Five 1-sec-long photostimulation cycles with 8-60 sec onset interval. The bar graph shows mean firing rate at each 50 ms-bin. The blue bar on the top indicates the duration of the photostimulation. We calculated mean firing rates of three phases (A: Cycle 1$)$ : the early phase $(0-0.5 \mathrm{sec}$ after the onset of photostimulation, blue), the late phase $(0.5-1 \mathrm{sec}$, red) and the post phase (1-1.5 sec, green) and summarized them in the right 3 plots. A. 8 - sec photostimulation interval ( $8 \mathrm{sec}$ cycle) (mean of 614 isolated neurons). B. 10 sec cycle ( $n=335)$. C. 20 sec cycle ( $n=692)$. D. 60 sec cycle $(n=139)$. 
Figure 4. Residual effect after intermission. A. Protocol for measuring the residual effect and the representative results of a 10 -sec and 30-sec intermission. Black vertical lines are individual spikes. B. Pre-intermission spike count difference (5th -1 st cycle) vs. post-intermission spike count difference (6th -1 st cycle). The spikes were counted for $4 \mathrm{sec}$ after the start of photostimulation in each cycle. The solid line is the identity line $(y=x)$, indicating a boundary of fully preserved residual effect. $\mathbf{C}$. Summary of the residual effect. The ratio of post-intermission spike count difference to pre-intermission spike count difference - the remnant. The error bar at each mean point represents SEM.

Figure 5. CNQX and AP5 bath-application altered the firing response pattern. Red lines indicate the control mean firing rate shown in Fig. 3C. The black dotted line in the right 3 plots indicates the control mean firing rate.

Figure 6. $\beta$-adrenergic receptor ( $\beta$-AR) modulations. A. $50 \mu \mathrm{M}$ isoproterenol, a $\beta$-AR agonist, increased the 1 st cycle early phase firing response $(n=61, p<0.05)$ and reduced the 1 st cycle post phase response $(n=61, p<0.05)$. Red lines indicate the control mean firing rate shown in Fig. 3C. B. $20 \mu \mathrm{M}$ adrenaline increased the 1 st cycle early phase firing response as well $(\mathrm{n}=35, \mathrm{p}<0.005)$. C. Applications of $20 \mu \mathrm{M}$ adrenaline with $20 \mu \mathrm{M}$ propranolol, a $\beta$-AR antagonist, did not show any significant enhancement of 1 st cycle early phase firing response $(n=39, p=0.75)$. 
Figure 7. $\alpha_{1}$-AR mediated modulation. A. 20 -sec cycle photostimulation with $30 \mu \mathrm{M}$ phenylephrine, an $\alpha_{1}$-AR agonist. Phenylephrine decreased the 1 st cycle early phase firing response $(n=92, p<0.01)$. The late and post phase firing increment between 1 st and 5th cycles was significantly increased $(n=92$, $\mathrm{p}<0.05$ in late phase, $\mathrm{p}<0.0005$ in post phase). Phenylephrine also increased the residual firings in the 5th cycle. B. $50 \mu \mathrm{M}$ noradrenaline decreased the 1 st cycle early phase firing response $(n=118$, $\mathrm{p}<0.0001)$.

Figure 8. Summary of the AR modulations to optogenetically induced firings. A. 1st cycle firing response in each phase. B. Firing increment in each phase. Asterisks indicate significant difference to control group. Error bars represent SEM. 


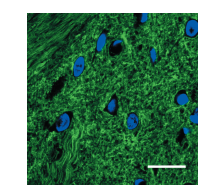

B
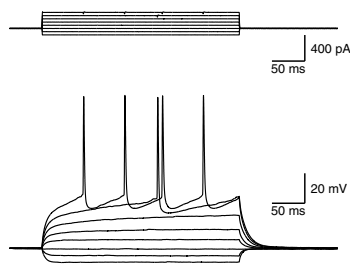

D

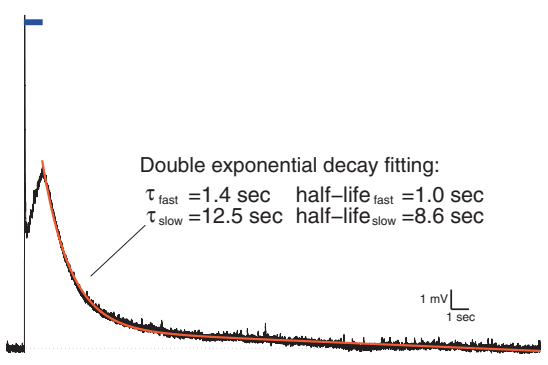

Cycle 1

Cycle 2

Cycle 3

Cycle 4

Cycle 5

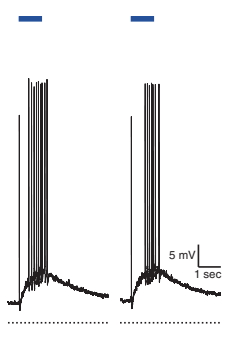

G Cycle 1

Cycle 2

Current injection
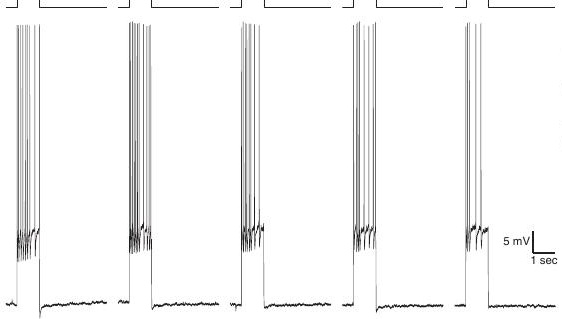

F

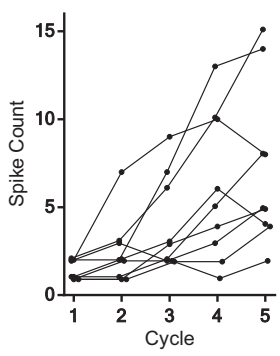

Cycle 3

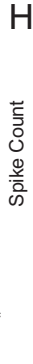

$\rightarrow 300 \mathrm{pA}$

... $250 \mathrm{pA}$

- $200 \mathrm{pA}$
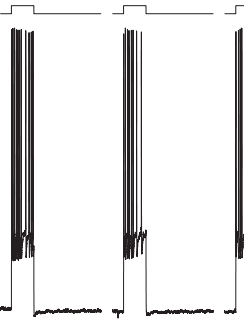

... $100 \mathrm{pA}$

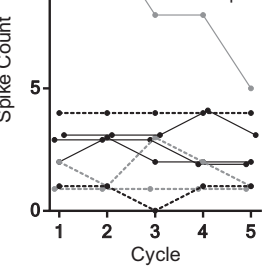


Figure 2
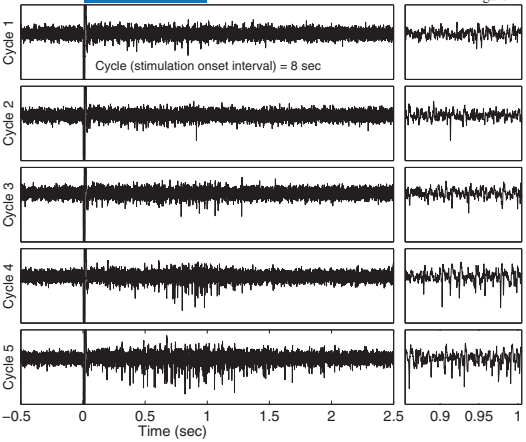


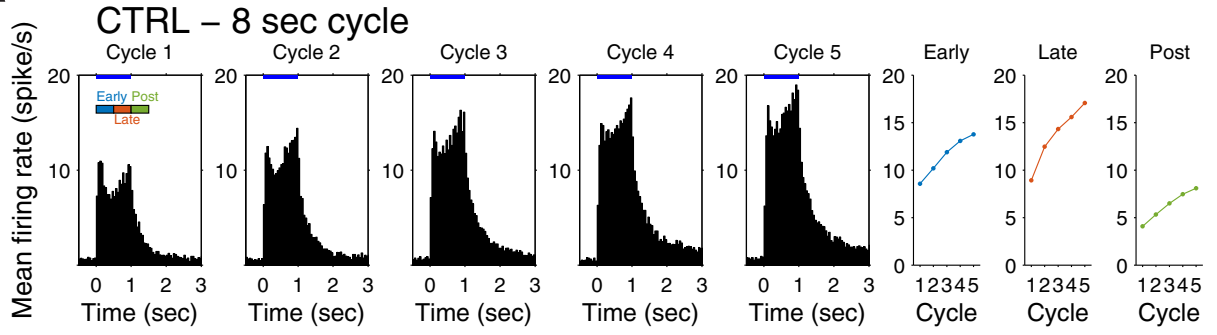

\section{(1) CTRL - 10 sec cycle}
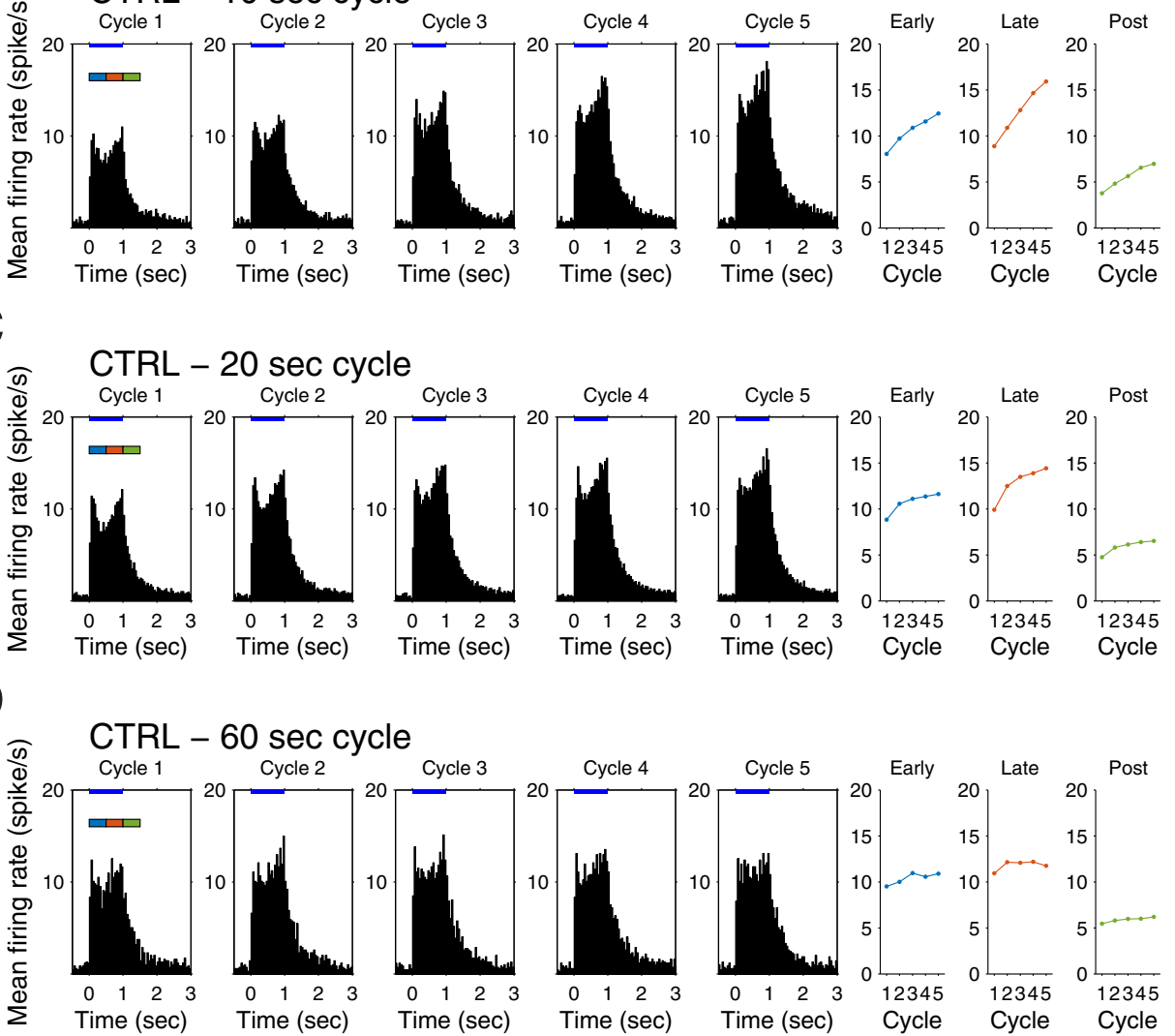
क Isoproterenol - $20 \mathrm{sec}$ cycle
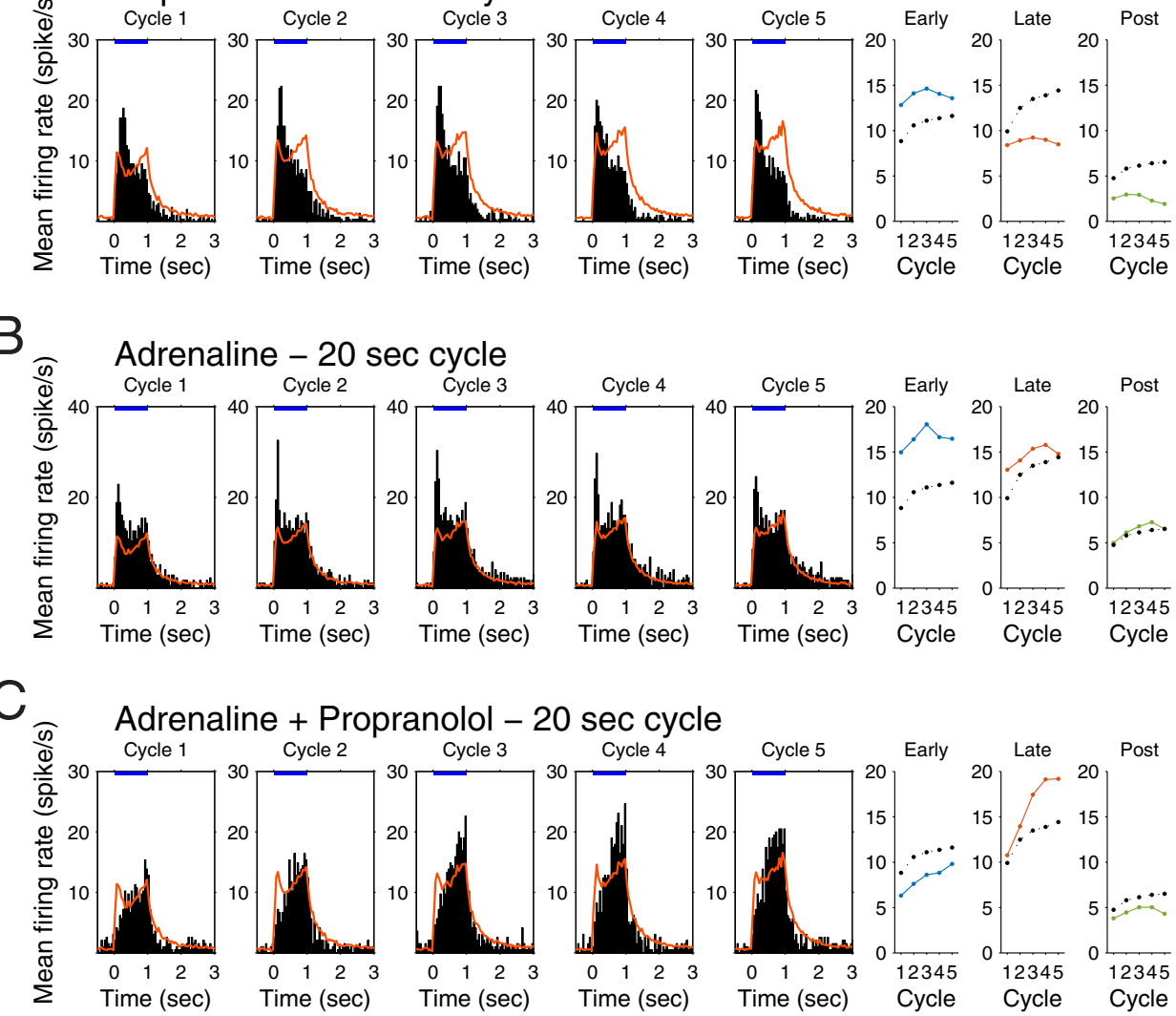


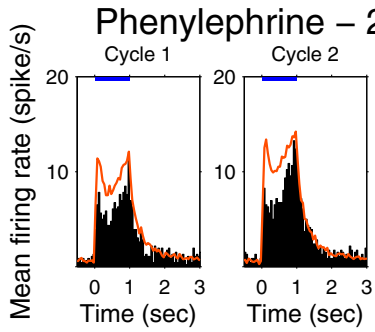

20 sec cycle
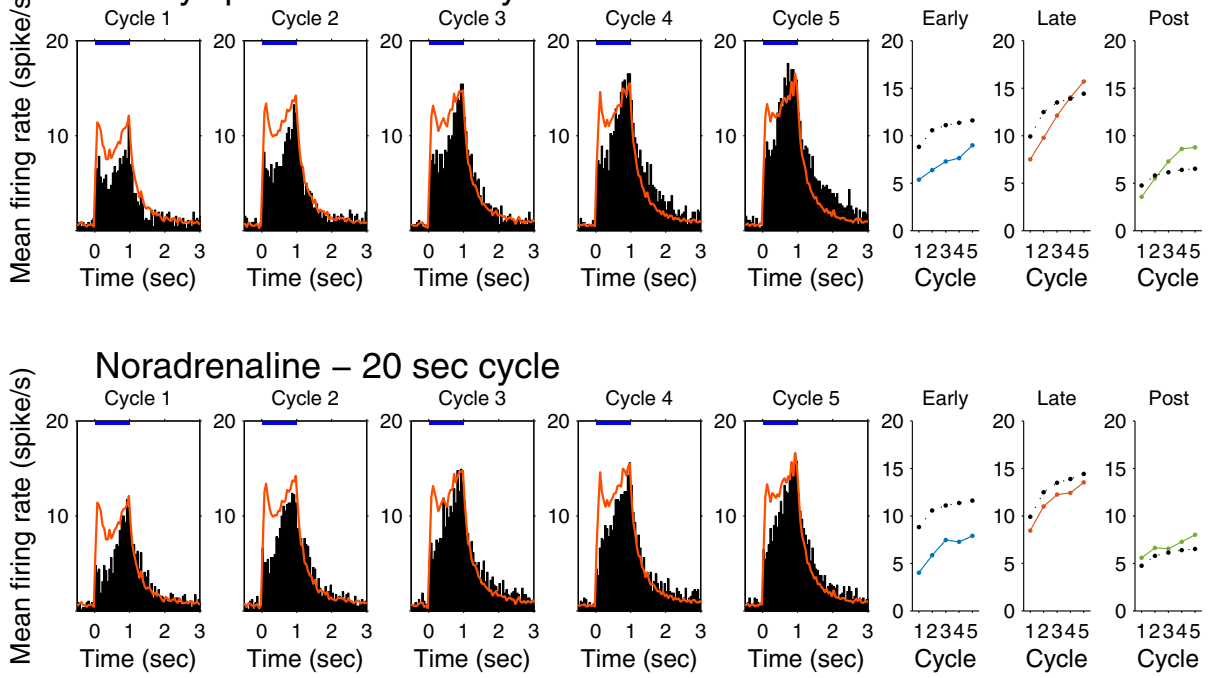
